\title{
Anal Cancer with Mediastinal Lymph Node Metastasis
}

\author{
Mangalore Amith Shenoy ${ }^{a}$ Lydia Winnicka $^{a}$ Leili Mirsadraei $^{b}$ Douglas Marks $^{c}$ \\ ${ }^{a}$ Department of Pulmonary and Critical Care, NYU Winthrop Hospital, Mineola, NY, USA; 'bepartment of Pathology, \\ NYU Winthrop Hospital, Mineola, NY, USA; 'Department of Hematology and Oncology, NYU Winthrop Hospital, \\ Mineola, NY, USA
}

\section{Keywords}

Anal cancer · Colorectal cancer · Metastasis · Imaging · Biopsy

\begin{abstract}
Squamous cell carcinoma of the anal canal remains rare, with metastatic disease even less commonly reported. We present a case of a patient with both a prior history of squamous cell carcinoma of the anal canal as well as breast cancer, who was without evidence of disease for 1 year. She was subsequently found to have FDG-avid mediastinal lymphadenopathy, initially assumed to be related to her more recent breast cancer. However, a biopsy confirmed recurrent anal cancer, with HPV infection. This represents a novel site of spread for anal cancer, one not yet reported in the literature.

\section{(C) 2021 The Author(s)}

Published by S. Karger AG, Basel
\end{abstract}

\section{Introduction}

Squamous cell carcinoma (SCC) of the anal canal is rare, though has a steadily increasing incidence over the last 2 decades, with a current incidence rate of 10.7-15.5 per 1,000,000 [1]. Distant metastatic spread is also rare, only reported in 15\% of cases [2]. Metastatic spread of anal SCC to the mediastinum, specifically to the mediastinal lymph nodes without lung involvement, has not yet

karger@karger.com www.karger.com/gat

Karger $\stackrel{\text { ' }}{=}$

BOPEN ACCESS
(C) 2021 The Author(s)

Published by S. Karger AG, Basel

This article is licensed under the Creative Commons AttributionNonCommercial-NoDerivatives 4.0 International License (CC BYNC-ND) (http://www.karger.com/Services/OpenAccessLicense). Usage and distribution for commercial purposes as well as any distribution of modified material requires written permission. been described in the literature. We present such a case, of a 76-year-old female, with a prior history of early-stage anal SCC and subsequent metastasis 2 years later to the mediastinal lymph nodes.

\section{Case Description}

A 74-year-old female initially presented to the pulmonary clinic with positron emission tomography (PET)positive mediastinal lymphadenopathy, for consideration of biopsy via endobronchial ultrasound. Her prior oncological history includes early-stage anal SCC that was initially discovered after she presented with BRBPR in May 2018. On subsequent colonoscopy, a 7-mm cratered ulcer was seen at the anus, with surrounding congested, erythematous, and nodular mucosa. A biopsy confirmed moderately-to-poorly differentiated SCC with smooth muscle invasion. Human papilloma virus (HPV) testing at that time is unknown. She was treated with the Nigro protocol (combined radiation and chemotherapy with 5-fluorouracil and mitomycin), which she completed in August 2018. Since then, she had been closely followed with surveillance CT scans with no evidence of recurrence or metastatic spread. However, she was subsequently diagnosed with stage 1A (T1bN0M0) left-sided breast cancer, discovered on routine screening mammogram and confirmed on breast biopsy in January 2019.

Lydia Winnicka

Department of Pulmonary and Critical Care, NYU Winthrop Hospital

222 Station Plaza North

Mineola, NY 11501 (USA)

lydiawinnicka@gmail.com 


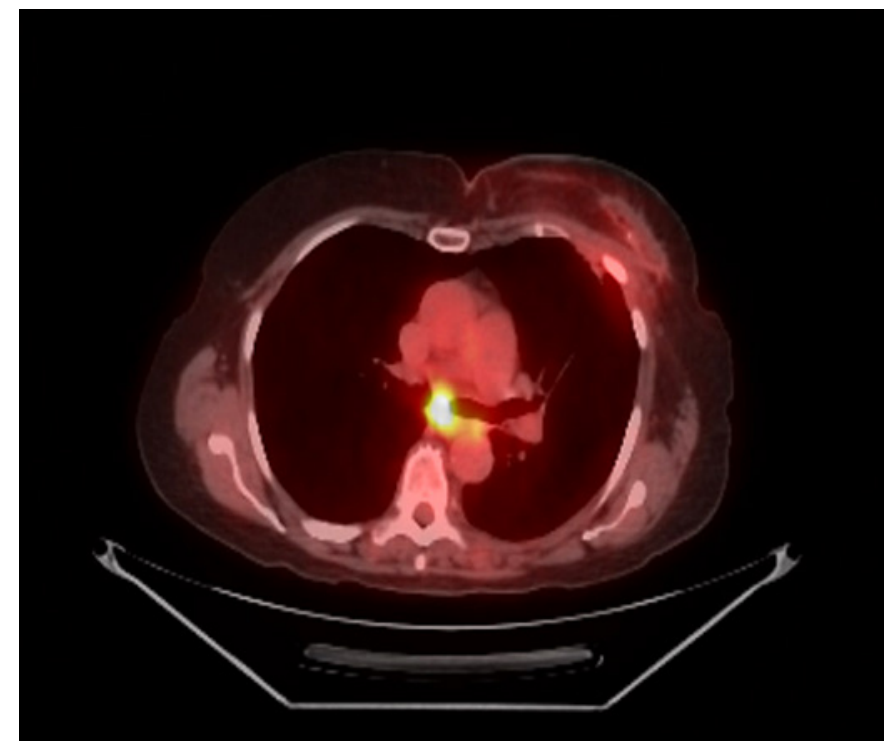

Fig. 1. Station 7 (subcarinal) FDG-avid lymphadenopathy. FDG, fluorodeoxyglucose.

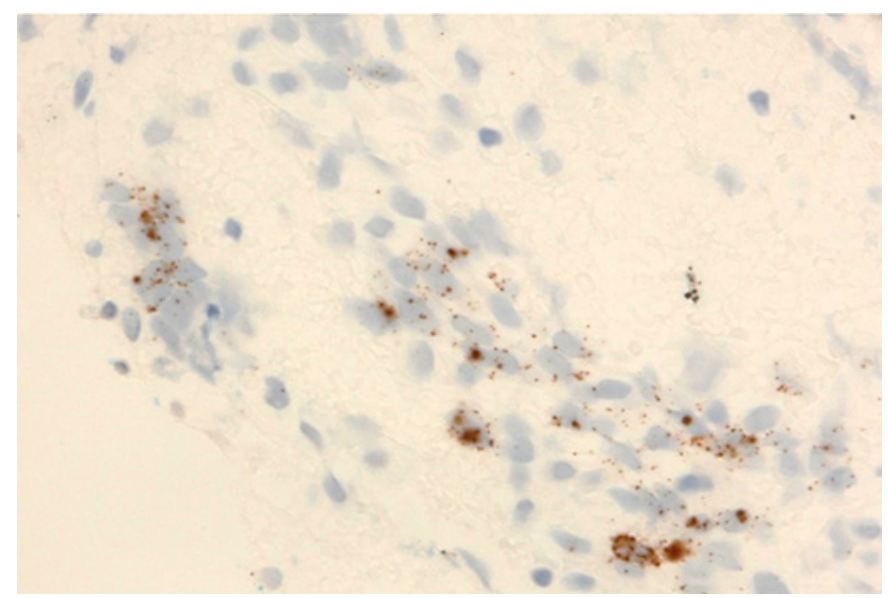

Fig. 3. Positive immunohistochemical stain for HPV. HPV, human papilloma virus.

Pathology showed ductal carcinoma with lobular features. Biomarker testing revealed the tumor to be ER positive, PR negative, and HER2 negative. She underwent a lumpectomy, adjuvant chemotherapy with 4 cycles of docetaxel and cyclophosphamide, and whole breast radiotherapy. She was then maintained on an aromatase inhibitor.

She had since been doing well with no active symptoms. In March 2020, she underwent a surveillance CT scan of the chest, abdomen, and pelvis, which revealed new mediastinal lymphadenopathy. New prevascular

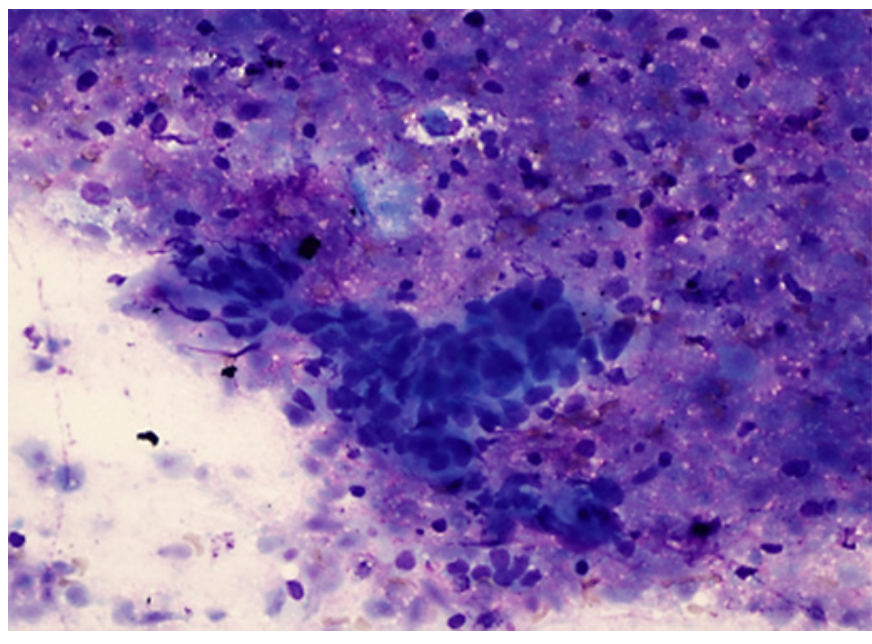

Fig. 2. Tightly cohesive clusters of squamous cells with nuclear pleomorphism in a necrotic background.

lymphadenopathy measuring $1.6 \times 1.0 \mathrm{~cm}$ and new subcarinal lymphadenopathy measuring $2.2 \times 1.6 \mathrm{~cm}$ were seen. No suspicious lung nodules were seen. A subsequent PET scan showed these lymph nodes to be fluorodeoxyglucose avid (prevascular, standard uptake value of 1.7; subcarinal, standard uptake value of 4.4) (Fig. 1). There were no other suspicious lesions. At that point, she was referred to the pulmonary clinic for a bronchoscopy with endobronchial ultrasound and biopsy. The procedure was performed in June 2020, with biopsies taken of station $4 \mathrm{~L}$ and 7 lymph nodes, both sites revealing SCC. Immunohistochemical stains performed on the tumor cells were positive for $\mathrm{P} 40, \mathrm{P} 63$, and CK5/6, weakly positive for GATA3 and ER, and negative for SOX10 and PR (Fig. 2). Tumor cells were also positive for HPV 16/18 (Fig. 3). Given her clinical history, these findings were felt to be consistent with metastatic anal SCC, albeit with an unusual metastatic pattern. She is currently planned to start chemotherapy with a platinum and taxane regimen.

\section{Discussion}

Anal cancer remains rare and accounts for only $1.5 \%$ of all gastrointestinal malignancies [3]. Usually presenting as a perianal mass with associated pain, bleeding, and pruritis, it may initially be mistaken for a benign condition, such as hemorrhoids. Clinical evaluation of a suspected anal cancer includes a physical exam with a digital rectal exam, visualization and biopsy of the anal mass via 
anoscopy, and imaging of the abdomen and pelvis with CT or MRI [3]. Anoscopy may reveal an obvious mass or condyloma or an area of abnormal mucosa, which may be raised or thickened [4]. Imaging can also help localize the mass and assess for any regional spread [3]. The anal canal, which begins at the anorectal ring and ends at the perianal skin, is divided by the dentate (or pectinate) line, which marks the transition from proximal glandular mucosa to distal squamous mucosa [3]. Histologically, anal cancers are thus subdivided into SCC and adenocarcinoma. Basaloid (or cloacogenic) carcinoma, a variant of SCC, arises from the epithelial transition zone and is nonkeratinizing, as opposed to other SCCs [3]. Importantly, anal cancer must also be distinguished from primary perianal skin cancer.

Approximately $70-90 \%$ of cases of anal cancer are related to strains of HPV [5]. Specifically, oncogenic subtypes of HPV include 16, 18, 31, 33, 45, 52, and 58 [6]. Other established risk factors include female gender, receptive anal intercourse, human immunodeficiency virus, smoking, and Crohn's disease [3]. Currently, CT of the chest, abdomen, and pelvis, MRI of the pelvis, and whole body PET-CT are recommended at the time of diagnosis to assess for locoregional spread and distant metastasis [1]. Anal cancer is typically spread via lymphatic drainage to the internal iliac lymph nodes in lesions above the pectinate line and to the superficial inguinal lymph nodes in lesions below the pectinate line [3, 7]. Metastatic disease, which remains very rare, typically has poor survival, with a 5 -year median survival rate of $10 \%$ in men and $20 \%$ in women. The liver followed by the lungs are the most common sites of spread [2]. Case reports of spread to the iris, brain, and cervical lymph nodes have also been described $[3,7]$. Our patient's PET scan was not helpful in determining the origin of her metastasis, as there was no new primary lesion. However, it is not uncommon for breast cancer to metastasize to the lungs and/or mediastinal lymph nodes. To determine the exact origin of spread, she underwent a mediastinal lymph node biopsy. While very rarely breast cancer may be of a squamous cell histology, her initial breast cancer was a different histological subtype, and her new biopsy showed HPV infection, both of which are more consistent with metastatic spread of anal cancer.

The Nigro protocol, first described in 1974, remains the first-line therapy for nonmetastatic anal cancer and includes 5-fluorouracil, mitomycin, and a course of radiation traditionally administered over $4-5$ weeks $[8,9]$. However, in the setting of advanced disease, the treatment protocols are significantly less efficacious and typi- cally have a short duration of clinical response. Per the 2020 National Comprehensive Cancer Network Clinical Practice Guidelines, carboplatin and paclitaxel (preferred), cisplatin and 5-fluorouracil, or 5-fluorouracil and oxaliplatin are potential treatment regimens [10]. Following progression on first-line metastatic therapy, immunotherapy with checkpoint inhibitors pembrolizumab and nivolumab is recommended [6]. Again, the importance of determining the origin of a patient's metastasis is highlighted here. In breast cancer, careful assessment of molecular markers is necessary to guide treatment. In a postmenopausal patient such as our patient, first-line therapy for metastatic hormone-receptor-positive, HER2-negative breast cancer would be endocrine therapy, with a cyclin-dependent kinase $4 / 6$ inhibitor plus an aromatase inhibitor [11].

Our patient's case is quite unusual in that she developed 2 unrelated malignancies, both early stage and both treated with definitive therapy at the time of diagnosis, with no signs of early treatment failure. Initially, she was diagnosed with early-stage anal SCC and was treated with the well-established Nigro protocol, detailed previously. She subsequently developed early-stage breast cancer, for which she underwent lumpectomy, followed by adjuvant chemotherapy, radiotherapy, and endocrine therapy. On subsequent imaging, done as part of anal cancer surveillance, she was found with fluorodeoxyglucose-avid mediastinal lymph nodes, which ultimately confirmed metastatic SCC with malignant cells demonstrating infection with high-risk HPV. This is a highly unusual site of distant metastasis and has not yet been described in the literature. With this case, we bring to attention a rare case of recurrence of anal cancer, which presented solely as mediastinal lymphadenopathy. Our case emphasizes the importance of a tissue biopsy to determine the cause of any new radiographic change, especially in a patient with a history of multiple malignancies.

\section{Conclusion}

SCC of the anal canal, though rare, has steadily increasing incidence. Metastatic spread is quite rare and has not yet been reported to the mediastinal lymph nodes. Our case thus illustrates a new and unusual metastatic site of anal SCC. Furthermore, as our patient also had a prior history of breast cancer, which admittedly was initially suspected to be the source of metastatic spread, we highlight the importance of prompt tissue biopsy in order to guide further treatment. 


\section{Statement of Ethics}

This study is exempt from ethics committee approval as it does not contain research data. Written informed consent was obtained from the patient for publication of this case report with accompanying images.

\section{Conflict of Interest Statement}

The authors have no conflicts of interest to disclose, financial or otherwise.

\section{Funding Sources}

No funding was used for this case report.

\section{Author Contributions}

Mangalore Amith Shenoy, MD: substantial contributions to the conception of the work, drafting the work and revising it critically for important intellectual content, and final approval of the version to be published. Lydia Winnicka, MD: substantial contributions to the conception of the work, drafting the work and revising it critically for important intellectual content, and final approval of the version to be published. Leili Mirsadraei, MD: substantial contributions to the conception of the work, drafting the work and revising it critically for important intellectual content, and final approval of the version to be published. Douglas Marks, MD: substantial contributions to the conception of the work, drafting the work and revising it critically for important intellectual content, and final approval of the version to be published.

\section{References}

1 Kin C. So now my patient has squamous cell cancer: diagnosis, staging, and treatment of squamous cell carcinoma of the anal canal and anal margin. Clin Colon Rectal Surg. 2018 Nov;31(6):353-60.

2 Dewdney A, Rao S. Metastatic squamous cell carcinoma of the anus: time for a shift in the treatment paradigm? ISRN Oncol. 2012 May; 2012:756591. Epub 2012 May 6.

3 Salati SA, Al Kadi A. Anal cancer: a review. Int J Health Sci. 2012 Jun;6(2):206-30.

4 Roberts JR, Siekas LL, Kaz AM. Anal intraepithelial neoplasia: a review of diagnosis and management. World J Gastrointest Oncol. 2017 Feb 15;9(2):50-61.
5 Martin D, Balermpas P, Winkelmann R, Rödel F, Rödel C, Fokas E. Anal squamous cell carcinoma: state of the art management and future perspectives. Cancer Treat Rev. 2018 Apr;65:11-21.

6 Morris VK, Eng C. Role of immunotherapy in the treatment of squamous cell carcinoma of the anal canal. J Natl Compr Canc Netw. 2018 Jul;16(7):903-8.

7 Wang B, Jaiswal S, Saif MW. First case of the cervical lymph node as the only site of metastasis from anal cancer. Cureus. 2017 May; 9(5):e1291.
8 Symer MM, Yeo HL. Recent advances in the management of anal cancer. F1000Res. 2018; 7:F1000. Faculty Rev-1572. Published online 2018 Sep 28.

9 Nigro ND, Vaitkevicius VK, Considine B Jr. Combined therapy for cancer of the anal canal: a preliminary report. Dis Colon Rectum. 1974;17(3):354-6.

10 Benson AB III, Arnoletti JP, Bekaii-Saab T. NCCN clinical practice guideline in oncology: anal carcinoma. NCCN.org. Accessed 2020 Jul 3.

11 Giordano SH, Elias AD, Gradishar WJ. NCCN guidelines updates: breast cancer. NCCN.org. Accessed 2020 Dec 6. 